\title{
Structure, caractéristiques et fonctionnement du réservoir aquifère des Grès Inférieurs du Trias en Lorraine
}

\author{
par Serge Bouly \\ de l'Association Scientifique pour la Géologie et ses Applications
}

et Laurent Demassieux

de l'Ecole Nationale Supérieure de Géologie

\section{I STRUCTURE DU RÉSERVOIR AQUI- FÈRE DES G.I.T.}

\subsection{Position stratigraphique}

Sur le plan stratigraphique, le réservoir aquifère des Grès Inférieurs du Trias se développe dans les assises gréseuses et conglomératiques du Buntsandstein, terme inférieur de la trilogie du Trias Germanique.

Il débute par les Grès d'Annweiler [1], équivalents des "Tigersandstein » de la Forêt Noire.

Il se poursuit par le Buntsandstein moyen composé généralement par un conglomérat de base sur quelques mètres puis par une imposante masse gréseuse pouvant atteindre $330 \mathrm{~m}$ et dénommée «Grès Vosgien » au sens strict.

\begin{abstract}
Le Grès Vosgien est surmonté par un niveau conglomératique puissant de 10 à $20 \mathrm{~m}$, le Conglomérat Principal (ou Poudingue de Sainte-Odile) formant un remarquable niveau repère. Dans certains secteurs, ce conglomérat est lui-même surmonté par un horizon violacé et bariolé, avec quelquefois des dolomies silicifiées et des nodules de cornaline auquel les Auteurs ont donné le nom de Zone Limite Violette.

Au-dessus apparaît le Buntsandstein supérieur composé de deux unités lithostratigraphiques : les couches intermédiaires et le Grès à Voltzia.

Ces couches intermédiaires sont constituées de bancs de grès lenticulaires brun-rouge avec niveaux sablo-argileux et argileux intercalés. Leur épaisseur est de 50 à $60 \mathrm{~m}$. Le Grès à Voltzia comprend des grès fins bien classés (le Grès à Meules), des grès à dalles, des bancs argileux et des bancs carbonatés peu épais. Le Grès à Voltzia se termine souvent, mais pas toujours, par un banc argileux rouge plus ou moins panaché de vert, épais de 1 à $2 \mathrm{~m}$ : l'Argile limite.
\end{abstract}

With regard to drinking and industrial water supply and to mining unwatering, the stratum of Lower Sandstones of the Trias in Lomaine is one of the most exploited aquifers in this area. The stratum is limited to the north and to the east by the outcrops of the Trias, to the west by an arbitrary line referring to a mine ralization rate above $I \mathrm{~g} /$, to the south by the Morvano-Vosgean Shelf.

The stratum is extending on hundreds of neters in depth between the crystalline formations of the base or: of the Permian argillaceous standstones and the shelly sandstone of the lower Muschelkalk.

The stratun is then confined and its flow rums in general south-north, still with some local variations almost due to collectings.

The reservoir shows a strong heterogeneity which can have a qualitative influence 
Classiquement, le toit du réservoir est considéré comme étant le grès coquillier du Muschelkalk inférieur. Ce faciès disparaît cependant pratiquement au droit du méridien de Nancy vers louest et aux environs de Rambervillers vers le sud [2].

La coupe suivante illustre la position du réservoir dans la série triasique.

\subsection{Structure du Trias Lorrain}

Les Grès Inférieurs du Trias sont affectés par de grands plis nord-est/sud-ouest [3]:
- les anticlinaux des Ardennes, du Hunsrück, Sarro-Lorrain, Vosges-Forêt Noire :

- les synclinaux du Luxembourg, de Neunkirchen et de Sarreguemines.

Des failles de même direction ou de direction orthogonale recoupent ces structures (cf. fig. 2). Des dômes anticlinaux ou des ennoyements d'axes synclinaux peuvent compliquer localement ces structures.

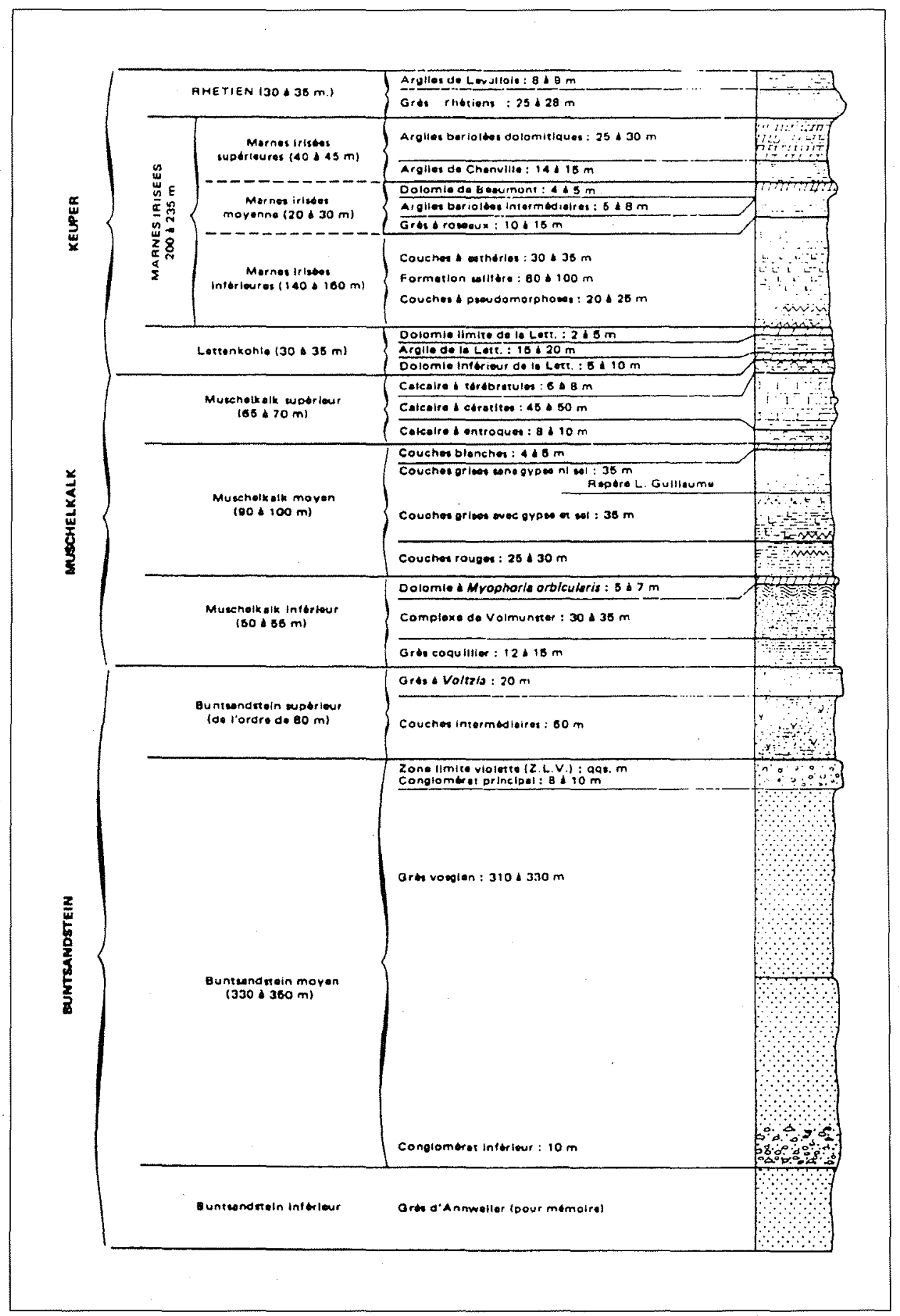

1. Coupe schématique des faciès lorrains dans l'axe synclinal de Sarreguemines (C. Mä̈aux). 


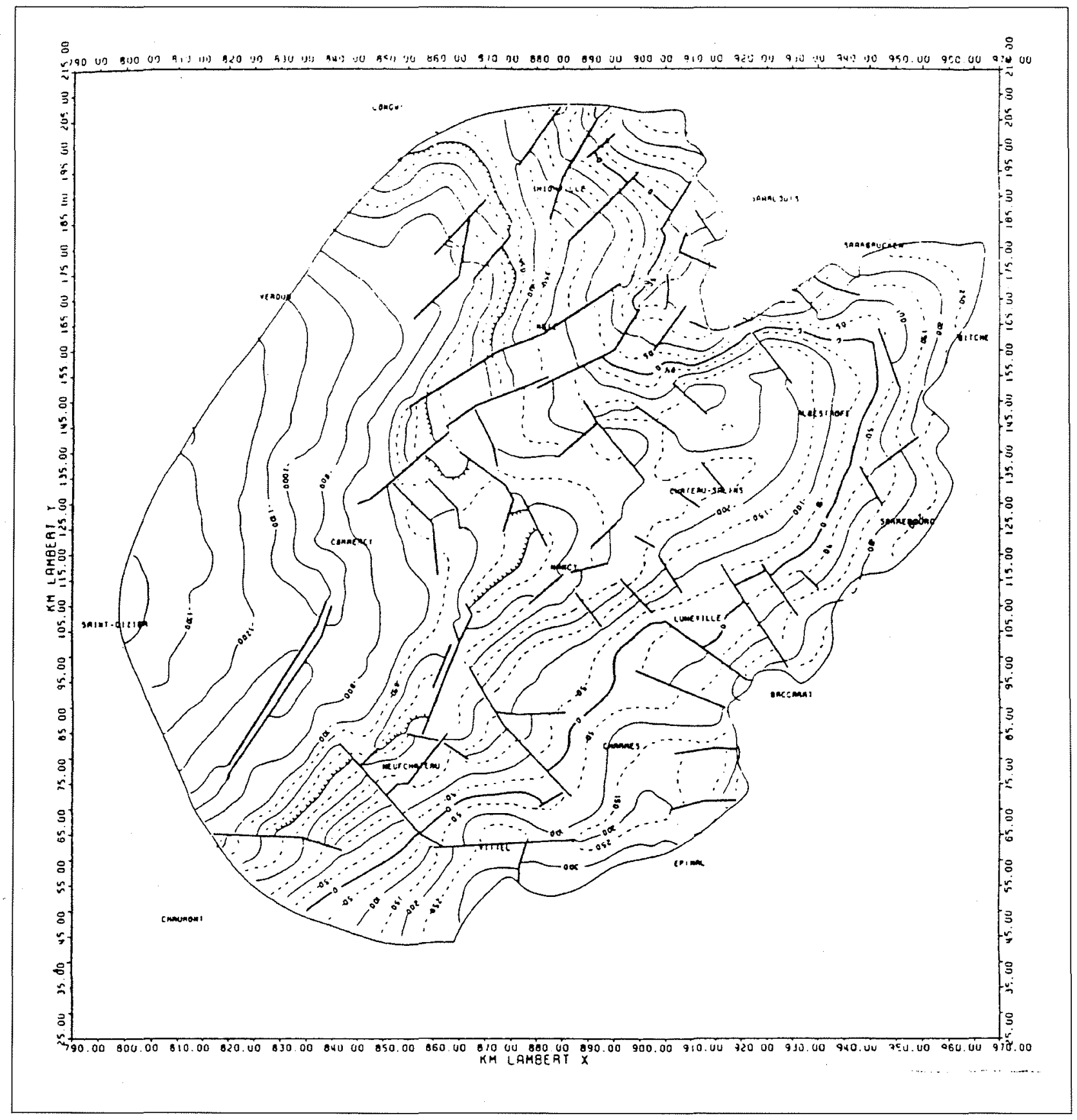

2. Carte structurale des Grès Inférieurs du Trias en Lorraine (J.J. Peraudin).

\section{FONCTIONNEMENT DE LA NAPPE AQUIFÈRE DES G.I.T.}

\subsection{Extension du réservoir aquifère}

Les limites horizontales correspondent au nord et au nord-est à la ligne d'érosion des affleurements gréseux, à l'est par la ligne de partage des eaux entre versants Lorrain et Alsacien des Vosges, et au sud par le seuil Morvano-Vosgien avec drainage de la nappe vers le bassin de la Saône. A l'ouest, nous devrions considérer comme limite l'extension des Grès à Voltzia, mais cette limite est en fait assez mal connue. De façon arbitraire, on admet une limite ouest d'exploitabilité aux fins
d'A.E.P. définie par un seuil de minéralisation à $1 \mathrm{~g} / \mathrm{l}$. Il ne s'agit bien entendu pas d'une limite au sens de l'hydrodynamisme.

Les limites verticales sont définies par les formations imperméables situées au mur et au toit des G.I.T. Au mur se pose le problème du Permien dont la nappe peut être en continuité hydraulique avec les G.I.T. Le toit sera considéré comme le sommet des grès coquilliers du Muschelkalk quand ils existent ou sinon le sommet des grès [3].

\section{龉 2.2 Alimentation et conditions aux limites}

Les zones d'alimentation de la nappe se situent au niveau des affleurements du Buntsandstein (Vosges et région houl- 


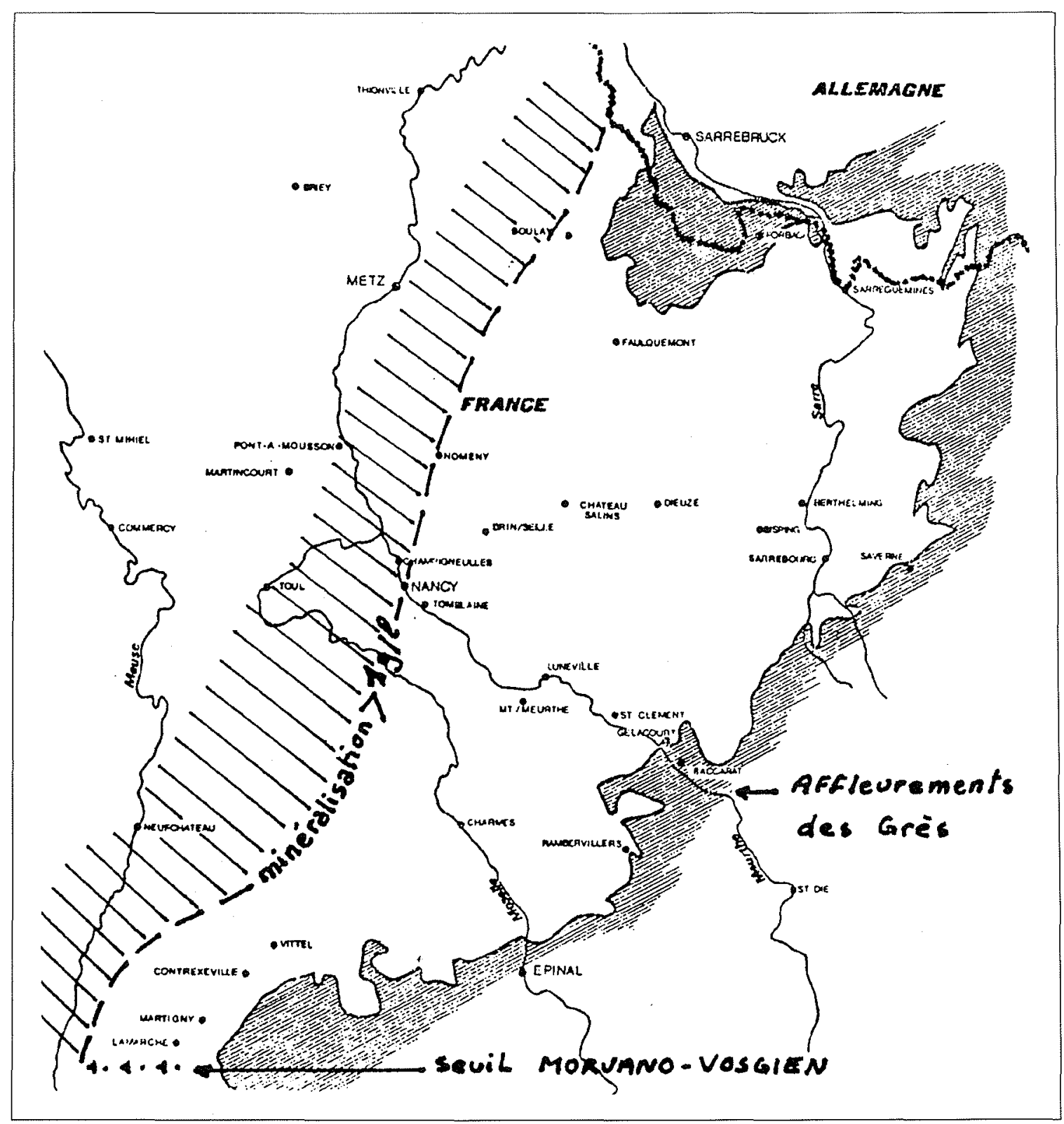

3. Limites du réservoir aquifère des G.I.T. en Lorraine.

lère principalement). La fraction infiltrée des précipitations ressort en partie par des sources ou par drainage par les ruisseaux. Une bonne partie alimente cependant la zone captive de la nappe. Les auteurs s'accordent à attribuer une valeur moyenne d'infiltration en zone d'affleurement de $8 \mathrm{l} / \mathrm{s} / \mathrm{km}^{2}$. Dans le secteur de Vittel, les affleurements participent peu à l'alimentation car la Saône et ses affluents drainent la nappe à contre pendage. Dans le secteur houiller, certains coefficients d'infiltration atteignent des valeurs fortes, de l'ordre de $30 \%$. Des échanges par drainance peuvent exister par ailleurs avec la nappe du Muschelkalk.

La nappe des Grès Inférieurs du Trias constitue la ressource en eau souterraine la plus importante de Lorraine. Ses réserves sont évaluées à 30 milliards de mètres cube en affleurement et 150 milliards de mètres cubes sous couverture.

\subsection{Exutoires de la nappe}

Le long des affleurements situés à l'est, le drainage s'effectue à contre pendage ( $c$ 'est le cas pour les vallées de l'Eichel, de la Zinsel, de la Sarre amont, de la Meurthe). Des «points de sortie » existent au niveau de la Moselle dans la fenêtre de Sierck-Lès-Bains et de la Sarre à l'est du bassin houiller.

Les importants rabattements provoqués au niveau du bassin houiller ont modifié les conditions initiales. Les vallées des rivières locales jouaient initialement un rôle de drain ; ce n'est plus vrai aujourd'hui.

Une réalimentation n'est pas à exclure à partir du Leisbach où se déversent les eaux d'exhaure de la Houve. Les failles majeures ont une incidence hydrodynamique probable. 
Pour assurer les besoins tant en eau potable qu'industrielle, ce sont plus de 150 millions de mètres cube qui sont prélevés annuellement.

\subsection{Piézométrie de la nappe}

Les mesures piézométriques sont souvent imprécises car la plupart des forages accessibles sont généralement exploités, interfèrent entre eux, et sont insuffisamment équipés (absence de manomètres, de tubes guide sonde, etc...).

Il est quasiment impossible d'obtenir des mesures synchrones compte tenu du nombre d'ouvrages à mesurer.

Malgré ces quelques problèmes « techniques », les états piézométriques font ressortir que la nappe des G.I.T. est très sollicitée et que les prélèvements y sont supérieurs aux apports depuis une trentaine d'années. Les potentialités de la nappe apparaissent ainsi menacées dans plusieurs secteurs :

- la région du Wandt - bassin houiller - Freyming-Merlebach,

- la région de Lunéville-Nancy,

- la région de Vittel-Contrexéville-Mirecourt.

Dans ces secteurs, des baisses importantes des niveaux piézométriques sont observées et il est à craindre une migration des eaux salées situées plus à l'ouest [4] ou des eaux sus-jacentes vers ces zones surexploitées.

La baisse piézométrique est en moyenne de $0,5 \mathrm{~m} / \mathrm{an}$ depuis 1950 et risque de s'accélérer si le parc de forages continue à crôtre à l'avenir.

A titre d'illustration de ces propos, nous présentons dans la figure 4 ci-après la piézométrie initiale calculée par modèle (J.J. Peraudin [3]) et l'esquisse piézométrique mesurée fin 1992 [5].

\section{CARACTÉRISTIQUES PRINCIPALES DE L'AQUIFÈRE DES G.I.T.}

\subsection{Transmissivité, perméabilité, coefficient d'emma- gasinement}

La transmissivité est variable d'un point à l'autre de la nappe mais une certaine répartition géographique peut se dégager. Ainsi, dans les secteurs à l'affleurement, dans plus de $75 \%$ des cas, la transmissivité est supérieure à $10^{-3} \mathrm{~m}^{2} / \mathrm{s}$. En secteur nord-est et sud-est, elle est généralement inférieure, de l'ordre de 4 à $7.10^{-4} \mathrm{~m}^{2} / \mathrm{s}$.

Dans le reste de la Lorraine, elle se situe généralement entre 2,5 et $5.10^{-3} \mathrm{~m}^{2} / \mathrm{s}$ avec quelquefois des valeurs plus importantes (Varangéville: $6,8 \cdot 10^{-3} \mathrm{~m}^{2} / \mathrm{s}$ ).

La perméabilité se situe dans une fourchette générale de $10^{-5}$ à $5.10^{-5} \mathrm{~m} / \mathrm{s}$

Le coefficient d'emmagasinement quant à lui est assez peu mesuré. Il est de l'ordre de $10^{-3}$ au droit de secteurs d'affleurements et de l'ordre de $10^{-4}$ sous couverture.

\subsection{Débits d'exploitation}

Les débits spécifiques varient dans une fourchette large de à $5 \mathrm{~m}^{3} / \mathrm{h} / \mathrm{m}$ et la plupart du temps entre 1 et $3 \mathrm{~m}^{3} / \mathrm{h} / \mathrm{m}$.

Les facteurs susceptibles d'influencer la répartition du débit spécifique des forages aux Grès Inférieurs du Trias sont [6]:

- l'épaisseur d'aquifère disponible ou capté ;

- les grands axes structuraux;

- en affleurement: la distance aux axes de drainage de la nappe ;
- les affaissements miniers dans le secteur du bassin houiller:

Les débits d'exploitation des ouvrages A.E.P. se situent la plupart du temps dans une fourchette de 60 à $100 \mathrm{~m}^{3} / \mathrm{h}$ mais certains ouvrages proches de Nancy peuvent débiter de 200 à $250 \mathrm{~m}^{3} / \mathrm{h}$. (Ils marquent fortement la piézométrie.)

\subsection{Tranche d'aquifère exploitée}

La quasi-totalité des ouvrages sont en fait des «puits imparfaits » qui ne captent pas l'ensemble de la série gréseuse. Il y a quelques décennies, les Grès à Voltzia et les couches intermédiaires étaient presque systématiquement captés. Ce n'est plus le cas car ces niveaux produisent généralement des eaux assez ferrugineuses. Les forages sont crépinés au droit du conglomérat principal et sur 80 à $90 \mathrm{~m}$ de « Grès Vosgien » au sens strict.

Capter plus profondément apporte peu de débit supplémentaire, les grès étant généralement beaucoup plus cimentés.

\subsection{Qualité des eaux}

Nous ne donnerons ici que quelques éléments sur la qualité des eaux de la nappe des G.I.T. qui comporte plus de 12000 analyses et qui a fait l'objet d'un premier travail sous forme de thèse de la part de V. Soukatchoff-Merrien [4]. Il est à retenir pour l'essentiel

- L'augmentation de la minéralisation avec la distance à l'affleurement.

- La faible minéralisation des eaux et son agressivité dans les secteurs d'affleurement avec ses conséquences en matière de santé publique (saturnisme hydrique par exemple).

- L'existence d'une «tache salée » avec forte minéralisation dans la région de Sarralbe.

- Des eaux sulfatées dans le bassin houiller pouvant traduire un apport d'eaux du Muschelkalk notamment par drainance.

- Des variations de la minéralisation en fonction de la structure.

- L'existence d'échanges de bases principalement au niveau de la frontière "nappe libre-nappe captive».

Récemment a été mise en évidence, dans le secteur nord Vittel-Mirecourt, l'existence d'arsenic à des teneurs dépassant la norme de $50 \mu \mathrm{g} / 1$ [7] [8]. L'hypothèse que nous avançons est qu'un apport d'eaux thermales profondes en provenance du socle est possible, une anomalie thermique ayant été constatée notamment au voisinage de la faille de Vittel.

Sur le plan bactériologique, les eaux sont de bonne qualité, ce qui fait que l'aquifère est de plus en plus sollicité notamment dans son secteur captif comme ressource de substitution de réservoirs plus vulnérables. Il apparait alors nécessaire aujourd'hui, compte tenu de la chute piézométrique continuellement mesurée, de gérer au mieux cette ressource de premier ordre pour la Lorraine.

Un modèle de gestion de la nappe est en cours de réalisation.

\section{Références}

[1] ForChe F. (1935). - Stratigraphie und Paläogeographie des Buntsandsteins im Unkreis der Vogesen. Mitteil. Geol. Staatinst. Hambourg, $n^{\circ} 15$, pp. 15-55, 9 fig

[2] Synthèse Géologique du Bassin de Paris. Stratigraphie et Paléogéographie. Mémoire du B.R.G.M. n 101. 


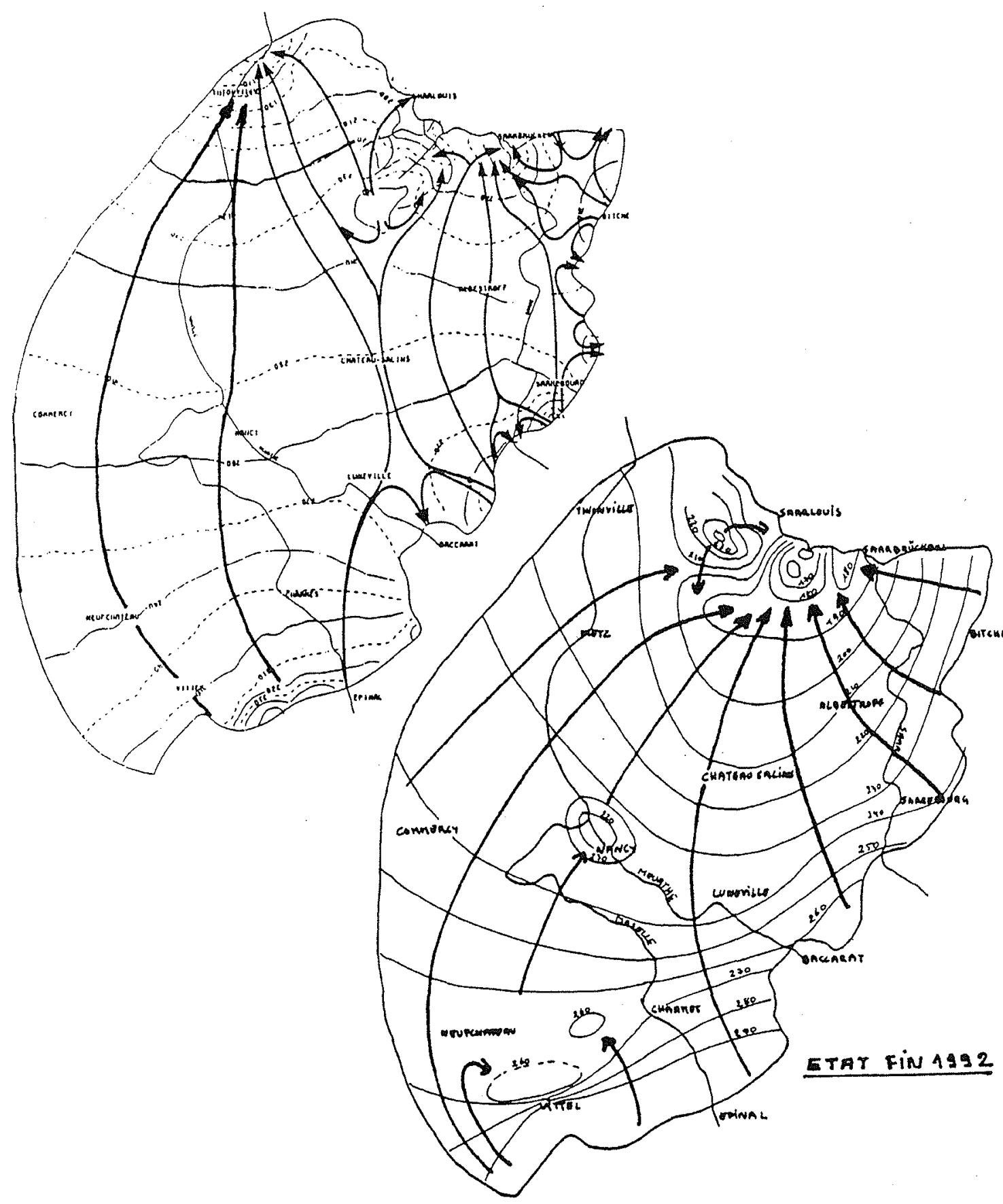

4. Piézométrie initiale calculée et piézométrie mesurée fin 1992.

[3] Peraudin J.J. (1983), - Conception et réalisation d'un modèle de gestion de la nappe des Grès Inférieurs du Trias en Lorraine. Thèse I.N.P.L./E.N.S.G.

[4] Soukatchoff-Merrien V. (1985). - Valorisation des fichiers qualité des eaux de la nappe des Grès Inférieurs du Trias. Thèse I.N.P.L.

[5] Bouly S. (1992). - Carle des isopièzes de la nappe des G.I.T. à $1 / 200000^{\circ}$ actualisée au $2^{\circ}$ trimestre 1992. Agence de l'Eau Rhin-Meuse.
[6] Ricour J. et Mathieu F. (1978). - Répartition statistique des débits spécifiques des forages exploitant la nappe des Grès du Trias Inférieur en Lorraine. Rapport 78 SGN 219 LOR. Ministère de l'Industrie, du Commerce et de l'Artisanat.

[7] Bouly S. (1989). - Syndicat des Eaux de la Vraine et du Xaintois. Réalisation d'un forage profond aux Grès Vosgiens à Baudricourt (88). Rapport hydrogéologique.

[8] Bouly S. (1994). - Syndicat des Eaux de la Vraine et du Xaintois. Réalisation d'un forage profond aux Grès Vosgiens à Saint-Menge (88). Rapport hydrogéologique. 